CRYSTALLOGRAPHIC COMMUNICATIONS

ISSN 2056-9890

Received 14 August 2017

Accepted 22 August 2017

Edited by P. C. Healy, Griffith University, Australia

Keywords: crystal structure; pyromellitic diimide; two-dimensional network; short S...S contact.

CCDC reference: 1570214

Supporting information: this article has supporting information at journals.iucr.org/e

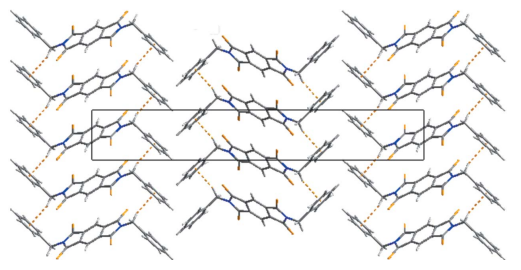

OPEN $\odot$ ACCESS

\section{Crystal structure of 2,6-dibenzylpyrrolo[3,4-f]- isoindole-1,3,5,7(2H,6H)-tetrathione}

\author{
Hansu Im, ${ }^{a}$ Hyunjin Park, ${ }^{\text {a Tae Ho Kim }}{ }^{\mathrm{a}}$ and Chang Hwa Woo ${ }^{\mathrm{b} *}$

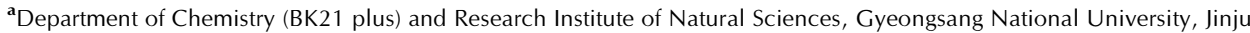 \\ 52828, Republic of Korea, and ${ }^{\mathbf{b}}$ Director of Planning Center, Gyeongsang National University Academy and Industry \\ Collaboration, 501 Jinjudaero, Jinjusi 52828, Republic of Korea. *Correspondence e-mail: woo@gnu.ac.kr
}

The title compound, $\mathrm{C}_{24} \mathrm{H}_{16} \mathrm{~N}_{2} \mathrm{~S}_{4}$, consists of a central pyromellitic diimide substituted with an $\mathrm{S}$ atom and terminal benzyl groups. The molecule lies on a crystallographic inversion centre so that the asymmetric unit contains half of the molecule. The molecule was prepared by thionation of $N, N^{\prime}$-dibenzylpyromellitic diimide with Lawesson's reagent and has an S-shaped conformation similar to other compounds of this type. The phenyl groups are tilted by $72.69(8)^{\circ}$ with respect to the plane of the central arene ring. In the crystal, molecules are connected by $\mathrm{C}-\mathrm{H} \cdots \pi$ interactions and weak short S $\cdots \mathrm{S}$ contacts, forming supramolecular layers extending paralled to the $a b$ plane. The crystal studied was found to be non-merohedrally twinned, with the minor component being 0.113 (3).

\section{Chemical context}

Recently, pyromellitic diimide derivatives have been spotlighted due to their use in energy-storage materials (Nalluri et al. 2016). They also show potential applications in photovoltaic devices (Kanosue et al., 2016) and organic semiconductors (Zheng et al., 2008). Not only pyromellitic diimide derivatives, but also pyromellitic diimides substituted with sulfur have potential applications in organic semiconductors (Yang et al., 2015). We have reported copper(I) coordination polymers based on pyromellitic diimide derivatives (Park et al., 2011), which showed colour change owing to intermolecular halogen $-\pi$ interactions. In addition, we have found that reversible solvent exchange and crystal transformations were possible in the crystals (Kang et al., 2015). In an extension of previous research, we have synthesized the pyromellitic diimide in which the $\mathrm{O}$ atoms are replaced with $\mathrm{S}$ atoms, by the reaction of $N, N^{\prime}$-dibenzylpyromellitic diimide with Lawesson's reagent, and report its crystal structure here.<smiles>S=c1c2cc3c(=S)n(Cc4ccccc4)c(=S)c3cc2c(=S)n1Cc1ccccc1</smiles>

\section{Structural commentary}

The molecular structure of the title compound consists of a central pyromellitic diimide substituted with $\mathrm{S}$ atoms and two terminal benzyl groups (Fig. 1). The molecule possesses a crystallographic inversion centre and thus the asymmetric unit 
Table 1

Hydrogen-bond geometry $\left(\AA,^{\circ}\right)$.

$C g 1$ is the centroid of the $\mathrm{C} 7-\mathrm{C} 12$ ring.

\begin{tabular}{lllll}
\hline$D-\mathrm{H} \cdots A$ & $D-\mathrm{H}$ & $\mathrm{H} \cdots A$ & $D \cdots A$ & $D-\mathrm{H} \cdots A$ \\
\hline $\mathrm{C} 6-\mathrm{H} 6 B \cdots \mathrm{S} 2$ & 0.99 & 2.75 & $3.208(3)$ & 109 \\
$\mathrm{C} 6-\mathrm{H} 6 B \cdots C g 1^{\mathrm{i}}$ & 0.99 & 2.66 & $3.498(3)$ & 142 \\
\hline
\end{tabular}

Symmetry code: (i) $x, y+1, z$.

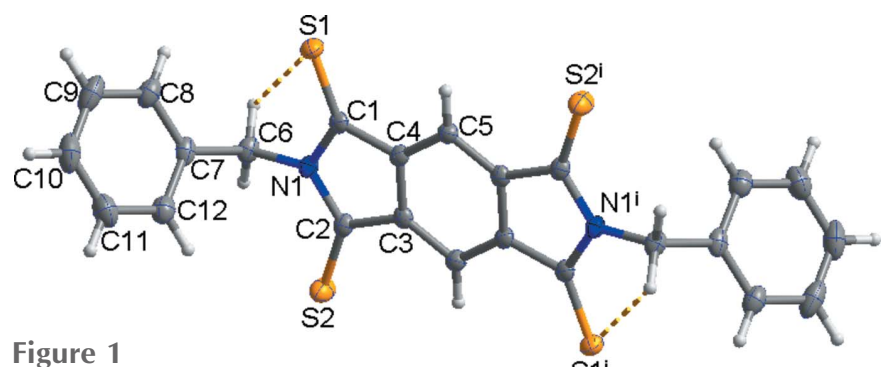

The asymmetric unit of the title compound, with 1 displacement ellipsoids drawn at the $50 \%$ probability level. $\mathrm{H}$ atoms are shown as small spheres of arbitrary radius and yellow dashed lines represent intramolecular $\mathrm{C}-$ $\mathrm{H} \cdots \mathrm{S}$ short contacts. Unlabelled atoms are generated by the symmetry operation $(-x+2,-y+1,-z)$.

of the title compound is composed of half a molecule. The molecule exhibits an intramolecular $\mathrm{C} 6-\mathrm{H} 6 B \cdots \mathrm{S} 2$ short contact (Table 1). In the molecule, the terminal phenyl groups point in opposite directions and their planes are tilted by $72.69(8)^{\circ}$ with respect to the plane of the central arene ring, forming an elongated S-shaped molecule.

\section{Supramolecular features}

In the crystal, $\mathrm{C} 6-\mathrm{H} 6 B \cdots C g 1^{\mathrm{i}}(C g 1$ is the centroid of the $\mathrm{C} 7-$ $\mathrm{C} 12$ ring) interactions between neighbouring molecules generate a one-dimensional loop chain (yellow dashed lines in Fig. 2, and Table 1). Moreover, adjacent molecules are connected by a weak short S1 . S S2 contact [3.5921 (10) $\AA$ ], resulting in the formation of a two-dimensional network (yellow and black dashed lines in Fig. 3).

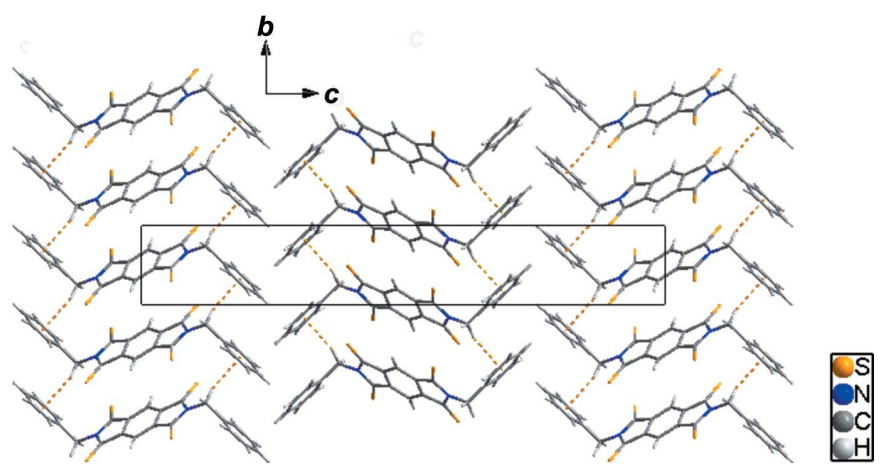

Figure 2

Intermolecular $\mathrm{C}-\mathrm{H} \cdots \pi$ interactions (yellow dashed lines) forming onedimensional loop chains.
Table 2

Experimental details.

\begin{tabular}{|c|c|}
\hline \multicolumn{2}{|l|}{ Crystal data } \\
\hline Chemical formula & $\mathrm{C}_{24} \mathrm{H}_{16} \mathrm{~N}_{2} \mathrm{~S}_{4}$ \\
\hline$M_{\mathrm{r}}$ & 460.63 \\
\hline Crystal system, space group & Monoclinic, $P 2_{1} / c$ \\
\hline Temperature $(\mathrm{K})$ & 173 \\
\hline$a, b, c(\AA)$ & $6.8571(4), 4.7724(3), 32.0010(17)$ \\
\hline$\beta\left(^{\circ}\right)$ & $95.916(4)$ \\
\hline$V\left(\mathrm{~A}^{3}\right)$ & $1041.65(11)$ \\
\hline$Z$ & 2 \\
\hline Radiation type & Мо $K \alpha$ \\
\hline$\mu\left(\mathrm{mm}^{-1}\right)$ & 0.47 \\
\hline Crystal size $(\mathrm{mm})$ & $0.43 \times 0.34 \times 0.01$ \\
\hline \multicolumn{2}{|l|}{ Data collection } \\
\hline Diffractometer & Bruker APEXII CCD \\
\hline Absorption correction & $\begin{array}{l}\text { Multi-scan (SADABS; Bruker, } \\
\text { 2014) }\end{array}$ \\
\hline$T_{\min }, T_{\max }$ & $0.646,0.746$ \\
\hline $\begin{array}{l}\text { No. of measured, independent and } \\
\text { observed }[I>2 \sigma(I)] \text { reflections }\end{array}$ & $1829,1829,1640$ \\
\hline$R_{\text {int }}$ & 0.059 \\
\hline$(\sin \theta / \lambda)_{\max }\left(\AA^{-1}\right)$ & 0.595 \\
\hline \multicolumn{2}{|l|}{ Refinement } \\
\hline$R\left[F^{2}>2 \sigma\left(F^{2}\right)\right], w R\left(F^{2}\right), S$ & $0.038,0.089,1.05$ \\
\hline No. of reflections & 1829 \\
\hline No. of parameters & 137 \\
\hline $\mathrm{H}$-atom treatment & $\mathrm{H}$-atom parameters constrained \\
\hline$\Delta \rho_{\max }, \Delta \rho_{\min }\left(\mathrm{e} \AA^{-3}\right)$ & $0.26,-0.23$ \\
\hline
\end{tabular}

Computer programs: APEX2 (Bruker, 2014), SAINT (Bruker, 2014), SHELXS97 (Sheldrick, 2008), SHELXL2014 (Sheldrick, 2015), DIAMOND (Brandenburg, 2010), SHELXTL (Sheldrick, 2008) and publCIF (Westrip, 2010).

\section{Synthesis and crystallization}

$N, N^{\prime}$-Dibenzylpyromellitic diimide was synthesized by the reaction of pyromellitic dianhydride with 2-phenylethylamine according to the literature procedure of Im et al. (2017). To a stirred solution of $N, N^{\prime}$-dibenzylpyromellitic diimide $(0.25 \mathrm{~g}$, $0.63 \mathrm{mmol})$ in anhydrous toluene $(100 \mathrm{ml})$ was added Lawesson's reagent $(2.00 \mathrm{~g}, 4.90 \mathrm{mmol})$, and the resulting mixture was stirred under reflux for $36 \mathrm{~h}$. It was then cooled to room temperature and concentrated in vacuo, followed by purification by silica-gel flash column chromatography $\left(\mathrm{CH}_{2} \mathrm{Cl}_{2}-n\right.$ hexane, 1:3 v/v). Crystals suitable for X-ray diffaction analysis were obtained by slow evaporation of a dichloromethane solution of the title compound.

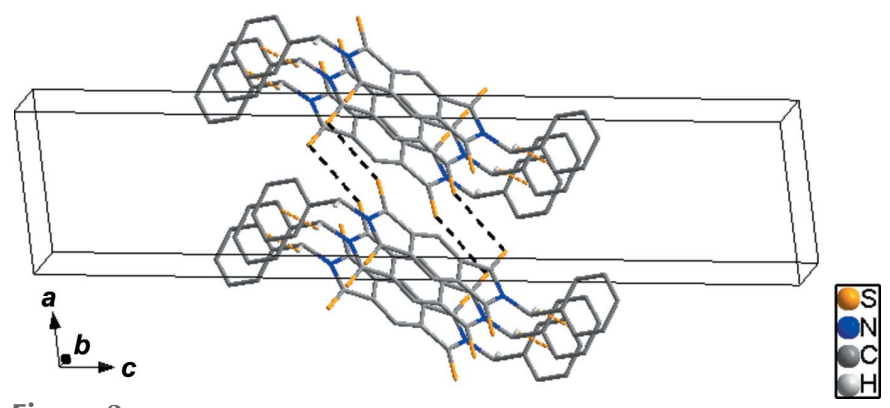

Figure 3

The packing diagram for the title compound, showing the twodimensional network formed by $\mathrm{C}-\mathrm{H} \cdots \pi$ interactions (yellow dashed lines) and weak short S ..S contacts (black dashed lines). H atoms not involved in intermolecular interactions have been omitted for clarity. 


\section{Refinement}

Crystal data, data collection and structure refinement details are summarized in Table 2. All $\mathrm{H}$ atoms were positioned geometrically and refined using a riding model, with $\mathrm{C}-\mathrm{H}=$ $0.95 \AA$ and $U_{\text {iso }}(\mathrm{H})=1.2 U_{\text {eq }}(\mathrm{C})$ for aromatic $\mathrm{C}-\mathrm{H}$ groups, and $\mathrm{C}-\mathrm{H}=0.99 \AA$ and $U_{\text {iso }}(\mathrm{H})=1.2 U_{\text {eq }}(\mathrm{C})$ for $\mathrm{Csp}^{3}-\mathrm{H}$ groups. Non-merohedral twinning was identified in the crystal (TwinRotMat within PLATON; Spek, 2009); the twin law is $-0.99900 .002,0-10,100.999$ and the final refined BASF parameter was determined to be 0.113 (3).

\section{Funding information}

Funding for this research was provided by: Ministry of Education, Science and Technology (Basic Science Program through the National Research Foundation of Korea (NRF); grant No. 2015R1D1A4A01020317); Ministry of Trade, Industry and Energy (MOTIE, Korea), Industrial Human Resources and Skill Development Program (award No. N0001415, Display Expert Training Project for Advanced Display equipments and components engineer).

\section{References}

Brandenburg, K. (2010). DIAMOND. Crystal Impact GbR, Bonn, Germany.

Bruker (2014). APEX2, SAINT and SADABS. Bruker AXS Inc., Madison, Wisconsin, USA.

Im, H., Choi, M. Y., Moon, C. J. \& Kim, T. H. (2017). Acta Cryst. E73, 838-841.

Kang, G., Jeon, Y., Lee, K. Y., Kim, J. \& Kim, T. H. (2015). Cryst. Growth Des. 15, 5183-5187.

Kanosue, K., Augulis, R., Peckus, D., Karpicz, R., Tamulevičus, T., Tamulevičius, S., Gulbinas, V. \& Ando, S. (2016). Macromolecules, 49, 1848-1857.

Nalluri, S. K. M., Liu, Z. C., Wu, Y. L., Hermann, K. R., Samanta, A., Kim, D. J., Krzyaniak, M. D., Wasielewski, M. R. \& Stoddart, J. F. (2016). J. Am. Chem. Soc. 138, 5968-5977.

Park, G., Yang, H., Kim, T. H. \& Kim, J. (2011). Inorg. Chem. 50, 961968.

Sheldrick, G. M. (2008). Acta Cryst. A64, 112-122.

Sheldrick, G. M. (2015). Acta Cryst. C71, 3-8.

Spek, A. L. (2009). Acta Cryst. D65, 148-155.

Westrip, S. P. (2010). J. Appl. Cryst. 43, 920-925.

Yang, T.-F., Huang, S.-H., Chiu, Y.-P., Chen, B.-H., Shih, Y.-W., Chang, Y.-C., Yao, J.-Y., Lee, Y.-J. \& Kuo, M.-Y. (2015). Chem. Commun. 51, 13772-13775.

Zheng, Q., Huang, J., Sarjeant, A. \& Katz, H. E. (2008). J. Am. Chem. Soc. 130, 14410-14411. 


\section{supporting information}

Acta Cryst. (2017). E73, 1379-1381 [https://doi.org/10.1107/S2056989017012154]

Crystal structure of 2,6-dibenzylpyrrolo[3,4-f] isoindole-1,3,5,7(2H,6H)-tetrathione

\section{Hansu Im, Hyunjin Park, Tae Ho Kim and Chang Hwa Woo}

Computing details

Data collection: APEX2 (Bruker, 2014); cell refinement: SAINT (Bruker, 2014); data reduction: SAINT (Bruker, 2014); program(s) used to solve structure: SHELXS97 (Sheldrick, 2008); program(s) used to refine structure: SHELXL2014 (Sheldrick, 2015); molecular graphics: DIAMOND (Brandenburg, 2010); software used to prepare material for publication: SHELXTL (Sheldrick, 2008) and publCIF (Westrip, 2010).

2,6-Dibenzylpyrrolo[3,4-f] isoindole-1,3,5,7(2H,6H)-tetrathione

\section{Crystal data}

$\mathrm{C}_{24} \mathrm{H}_{16} \mathrm{~N}_{2} \mathrm{~S}_{4}$

$M_{r}=460.63$

Monoclinic, $P 2{ }_{1} / c$

$a=6.8571$ (4) $\AA$

$b=4.7724(3) \AA$

$c=32.0010(17) \AA$

$\beta=95.916(4)^{\circ}$

$V=1041.65(11) \AA^{3}$

$Z=2$

\section{Data collection}

Bruker APEXII CCD diffractometer

$\varphi$ and $\omega$ scans

Absorption correction: multi-scan

(SADABS; Bruker, 2014)

$T_{\min }=0.646, T_{\max }=0.746$

1829 measured reflections

\section{Refinement}

Refinement on $F^{2}$

Least-squares matrix: full

$R\left[F^{2}>2 \sigma\left(F^{2}\right)\right]=0.038$

$w R\left(F^{2}\right)=0.089$

$S=1.05$

1829 reflections

137 parameters

0 restraints
$F(000)=476$

$D_{\mathrm{x}}=1.469 \mathrm{Mg} \mathrm{m}^{-3}$

Mo $K \alpha$ radiation, $\lambda=0.71073 \AA$

Cell parameters from 3915 reflections

$\theta=2.6-25.5^{\circ}$

$\mu=0.47 \mathrm{~mm}^{-1}$

$T=173 \mathrm{~K}$

Plate, brown

$0.43 \times 0.34 \times 0.01 \mathrm{~mm}$

1829 independent reflections

1640 reflections with $I>2 \sigma(I)$

$R_{\text {int }}=0.059$

$\theta_{\max }=25.0^{\circ}, \theta_{\min }=1.3^{\circ}$

$h=-8 \rightarrow 8$

$k=-5 \rightarrow 5$

$l=-6 \rightarrow 38$

Hydrogen site location: inferred from neighbouring sites

$\mathrm{H}$-atom parameters constrained

$w=1 /\left[\sigma^{2}\left(F_{\mathrm{o}}{ }^{2}\right)+(0.037 P)^{2}+0.6197 P\right]$ where $P=\left(F_{\mathrm{o}}^{2}+2 F_{\mathrm{c}}^{2}\right) / 3$

$(\Delta / \sigma)_{\max }<0.001$

$\Delta \rho_{\max }=0.26 \mathrm{e} \AA^{-3}$

$\Delta \rho_{\min }=-0.23 \mathrm{e} \AA^{-3}$ 


\section{Special details}

Geometry. All esds (except the esd in the dihedral angle between two 1.s. planes) are estimated using the full covariance matrix. The cell esds are taken into account individually in the estimation of esds in distances, angles and torsion angles; correlations between esds in cell parameters are only used when they are defined by crystal symmetry. An approximate (isotropic) treatment of cell esds is used for estimating esds involving l.s. planes.

Refinement. Refined as a 2-component twin

Fractional atomic coordinates and isotropic or equivalent isotropic displacement parameters $\left(\AA^{2}\right)$

\begin{tabular}{lllll}
\hline & $x$ & $y$ & $z$ & $U_{\text {iso }}{ }^{*} U_{\text {eq }}$ \\
\hline S1 & $0.50612(10)$ & $0.30438(16)$ & $0.05735(2)$ & $0.0259(2)$ \\
S2 & $1.10897(11)$ & $0.99091(17)$ & $0.10558(2)$ & $0.0299(2)$ \\
N1 & $0.7913(3)$ & $0.6607(5)$ & $0.08677(6)$ & $0.0192(5)$ \\
C1 & $0.7132(4)$ & $0.4707(6)$ & $0.05665(7)$ & $0.0189(6)$ \\
C2 & $0.9678(4)$ & $0.7718(6)$ & $0.07722(7)$ & $0.0199(6)$ \\
C3 & $1.0046(4)$ & $0.6467(6)$ & $0.03656(7)$ & $0.0182(6)$ \\
C4 & $0.8521(4)$ & $0.4592(6)$ & $0.02468(7)$ & $0.0184(6)$ \\
C5 & $1.1572(4)$ & $0.6945(6)$ & $0.01231(7)$ & $0.0199(6)$ \\
H5 & 1.2604 & 0.8225 & 0.0204 & $0.024^{*}$ \\
C6 & $0.6888(4)$ & $0.7471(6)$ & $0.12291(8)$ & $0.0232(6)$ \\
H6A & 0.5503 & 0.7888 & 0.1130 & $0.028^{*}$ \\
H6B & 0.7493 & 0.9218 & 0.1348 & $0.028^{*}$ \\
C7 & $0.6944(4)$ & $0.5277(6)$ & $0.15724(8)$ & $0.0235(6)$ \\
C8 & $0.5208(5)$ & $0.4058(6)$ & $0.16745(9)$ & $0.0300(7)$ \\
H8 & 0.3993 & 0.4576 & 0.1525 & $0.036^{*}$ \\
C9 & $0.5252(5)$ & $0.2086(7)$ & $0.19950(10)$ & $0.0411(9)$ \\
H9 & 0.4069 & 0.1252 & 0.2064 & $0.049^{*}$ \\
C10 & $0.7023(5)$ & $0.1345(7)$ & $0.22125(9)$ & $0.0423(9)$ \\
H10 & 0.7052 & 0.0004 & 0.2433 & $0.051^{*}$ \\
C11 & $0.8741(5)$ & $0.2531(7)$ & $0.21127(9)$ & $0.0383(8)$ \\
H11 & 0.9952 & 0.2000 & 0.2263 & $0.046^{*}$ \\
C12 & $0.8711(4)$ & $0.4503(6)$ & $0.17935(8)$ & $0.0289(7)$ \\
H12 & 0.9901 & 0.5326 & 0.1726 & $0.035^{*}$ \\
& & & & \\
\hline
\end{tabular}

Atomic displacement parameters $\left(\AA^{2}\right)$

\begin{tabular}{lllllll}
\hline & $U^{11}$ & $U^{22}$ & $U^{33}$ & $U^{12}$ & $U^{13}$ & $U^{23}$ \\
\hline S1 & $0.0227(3)$ & $0.0291(4)$ & $0.0261(3)$ & $-0.0078(3)$ & $0.0038(3)$ & $0.0008(3)$ \\
S2 & $0.0311(4)$ & $0.0301(5)$ & $0.0291(4)$ & $-0.0100(3)$ & $0.0063(3)$ & $-0.0092(3)$ \\
N1 & $0.0213(11)$ & $0.0168(13)$ & $0.0198(10)$ & $-0.0010(10)$ & $0.0041(9)$ & $0.0010(9)$ \\
C1 & $0.0212(13)$ & $0.0160(15)$ & $0.0193(12)$ & $0.0008(11)$ & $0.0013(10)$ & $0.0051(11)$ \\
C2 & $0.0235(14)$ & $0.0158(16)$ & $0.0210(12)$ & $0.0014(12)$ & $0.0046(10)$ & $0.0024(11)$ \\
C3 & $0.0208(13)$ & $0.0153(15)$ & $0.0184(12)$ & $0.0035(12)$ & $0.0022(10)$ & $0.0026(11)$ \\
C4 & $0.0196(13)$ & $0.0156(16)$ & $0.0202(12)$ & $-0.0001(11)$ & $0.0024(10)$ & $0.0046(11)$ \\
C5 & $0.0208(13)$ & $0.0177(16)$ & $0.0209(13)$ & $-0.0005(12)$ & $0.0012(10)$ & $0.0021(11)$ \\
C6 & $0.0279(14)$ & $0.0180(17)$ & $0.0249(13)$ & $0.0020(12)$ & $0.0089(11)$ & $-0.0020(12)$ \\
C7 & $0.0359(16)$ & $0.0163(16)$ & $0.0197(12)$ & $-0.0004(13)$ & $0.0094(11)$ & $-0.0036(11)$ \\
C8 & $0.0359(16)$ & $0.0259(19)$ & $0.0304(15)$ & $-0.0014(14)$ & $0.0145(13)$ & $-0.0022(13)$
\end{tabular}




\begin{tabular}{lllllll} 
C9 & $0.057(2)$ & $0.029(2)$ & $0.0427(17)$ & $-0.0066(17)$ & $0.0301(16)$ & $-0.0013(15)$ \\
C10 & $0.070(2)$ & $0.032(2)$ & $0.0276(16)$ & $-0.0005(18)$ & $0.0169(16)$ & $0.0040(14)$ \\
C11 & $0.053(2)$ & $0.033(2)$ & $0.0273(15)$ & $0.0034(17)$ & $0.0005(14)$ & $0.0030(14)$ \\
C12 & $0.0365(16)$ & $0.0238(18)$ & $0.0272(14)$ & $-0.0037(14)$ & $0.0068(12)$ & $-0.0010(13)$ \\
\hline
\end{tabular}

Geometric parameters $\left(\hat{A},{ }^{\circ}\right)$

\begin{tabular}{|c|c|c|c|}
\hline $\mathrm{S} 1-\mathrm{C} 1$ & $1.629(3)$ & $\mathrm{C} 6-\mathrm{H} 6 \mathrm{~A}$ & 0.9900 \\
\hline $\mathrm{S} 2-\mathrm{C} 2$ & $1.635(3)$ & C6-H6B & 0.9900 \\
\hline $\mathrm{N} 1-\mathrm{C} 2$ & $1.384(3)$ & $\mathrm{C} 7-\mathrm{C} 12$ & $1.388(4)$ \\
\hline $\mathrm{N} 1-\mathrm{C} 1$ & $1.390(3)$ & $\mathrm{C} 7-\mathrm{C} 8$ & $1.394(4)$ \\
\hline $\mathrm{N} 1-\mathrm{C} 6$ & $1.473(3)$ & $\mathrm{C} 8-\mathrm{C} 9$ & $1.390(4)$ \\
\hline $\mathrm{C} 1-\mathrm{C} 4$ & $1.469(3)$ & $\mathrm{C} 8-\mathrm{H} 8$ & 0.9500 \\
\hline $\mathrm{C} 2-\mathrm{C} 3$ & $1.477(3)$ & $\mathrm{C} 9-\mathrm{C} 10$ & $1.382(5)$ \\
\hline $\mathrm{C} 3-\mathrm{C} 5$ & $1.384(4)$ & C9- $\mathrm{H} 9$ & 0.9500 \\
\hline $\mathrm{C} 3-\mathrm{C} 4$ & $1.399(4)$ & $\mathrm{C} 10-\mathrm{C} 11$ & $1.374(5)$ \\
\hline $\mathrm{C} 4-\mathrm{C}^{\mathrm{i}}$ & $1.388(3)$ & $\mathrm{C} 10-\mathrm{H} 10$ & 0.9500 \\
\hline $\mathrm{C} 5-\mathrm{C}^{\mathrm{i}}$ & $1.388(3)$ & $\mathrm{C} 11-\mathrm{C} 12$ & $1.388(4)$ \\
\hline $\mathrm{C} 5-\mathrm{H} 5$ & 0.9500 & C11-H11 & 0.9500 \\
\hline $\mathrm{C} 6-\mathrm{C} 7$ & $1.515(4)$ & $\mathrm{C} 12-\mathrm{H} 12$ & 0.9500 \\
\hline $\mathrm{C} 2-\mathrm{N} 1-\mathrm{C} 1$ & $112.3(2)$ & $\mathrm{N} 1-\mathrm{C} 6-\mathrm{H} 6 \mathrm{~B}$ & 108.9 \\
\hline $\mathrm{C} 2-\mathrm{N} 1-\mathrm{C} 6$ & $124.4(2)$ & $\mathrm{C} 7-\mathrm{C} 6-\mathrm{H} 6 \mathrm{~B}$ & 108.9 \\
\hline $\mathrm{C} 1-\mathrm{N} 1-\mathrm{C} 6$ & $123.1(2)$ & $\mathrm{H} 6 \mathrm{~A}-\mathrm{C} 6-\mathrm{H} 6 \mathrm{~B}$ & 107.7 \\
\hline $\mathrm{N} 1-\mathrm{C} 1-\mathrm{C} 4$ & $106.0(2)$ & $\mathrm{C} 12-\mathrm{C} 7-\mathrm{C} 8$ & $119.4(3)$ \\
\hline $\mathrm{N} 1-\mathrm{C} 1-\mathrm{S} 1$ & $125.63(19)$ & $\mathrm{C} 12-\mathrm{C} 7-\mathrm{C} 6$ & $120.6(2)$ \\
\hline $\mathrm{C} 4-\mathrm{C} 1-\mathrm{S} 1$ & $128.3(2)$ & $\mathrm{C} 8-\mathrm{C} 7-\mathrm{C} 6$ & $120.0(2)$ \\
\hline $\mathrm{N} 1-\mathrm{C} 2-\mathrm{C} 3$ & $105.8(2)$ & $\mathrm{C} 9-\mathrm{C} 8-\mathrm{C} 7$ & $120.1(3)$ \\
\hline $\mathrm{N} 1-\mathrm{C} 2-\mathrm{S} 2$ & $127.14(19)$ & $\mathrm{C} 9-\mathrm{C} 8-\mathrm{H} 8$ & 120.0 \\
\hline $\mathrm{C} 3-\mathrm{C} 2-\mathrm{S} 2$ & $127.06(19)$ & $\mathrm{C} 7-\mathrm{C} 8-\mathrm{H} 8$ & 120.0 \\
\hline $\mathrm{C} 5-\mathrm{C} 3-\mathrm{C} 4$ & $122.7(2)$ & $\mathrm{C} 10-\mathrm{C} 9-\mathrm{C} 8$ & $119.7(3)$ \\
\hline $\mathrm{C} 5-\mathrm{C} 3-\mathrm{C} 2$ & $129.4(2)$ & $\mathrm{C} 10-\mathrm{C} 9-\mathrm{H} 9$ & 120.1 \\
\hline $\mathrm{C} 4-\mathrm{C} 3-\mathrm{C} 2$ & $107.9(2)$ & $\mathrm{C} 8-\mathrm{C} 9-\mathrm{H} 9$ & 120.1 \\
\hline $\mathrm{C} 5^{\mathrm{i}-\mathrm{C}} 4-\mathrm{C} 3$ & $122.5(2)$ & $\mathrm{C} 11-\mathrm{C} 10-\mathrm{C} 9$ & $120.5(3)$ \\
\hline $\mathrm{C} 5^{\mathrm{i}}-\mathrm{C} 4-\mathrm{C} 1$ & $129.6(2)$ & $\mathrm{C} 11-\mathrm{C} 10-\mathrm{H} 10$ & 119.8 \\
\hline $\mathrm{C} 3-\mathrm{C} 4-\mathrm{C} 1$ & $107.9(2)$ & $\mathrm{C} 9-\mathrm{C} 10-\mathrm{H} 10$ & 119.8 \\
\hline $\mathrm{C} 3-\mathrm{C} 5-\mathrm{C} 4^{\mathrm{i}}$ & $114.8(2)$ & $\mathrm{C} 10-\mathrm{C} 11-\mathrm{C} 12$ & $120.2(3)$ \\
\hline $\mathrm{C} 3-\mathrm{C} 5-\mathrm{H} 5$ & 122.6 & $\mathrm{C} 10-\mathrm{C} 11-\mathrm{H} 11$ & 119.9 \\
\hline $\mathrm{C} 4-\mathrm{C} 5-\mathrm{H} 5$ & 122.6 & $\mathrm{C} 12-\mathrm{C} 11-\mathrm{H} 11$ & 119.9 \\
\hline $\mathrm{N} 1-\mathrm{C} 6-\mathrm{C} 7$ & $113.4(2)$ & $\mathrm{C} 11-\mathrm{C} 12-\mathrm{C} 7$ & $120.1(3)$ \\
\hline $\mathrm{N} 1-\mathrm{C} 6-\mathrm{H} 6 \mathrm{~A}$ & 108.9 & $\mathrm{C} 11-\mathrm{C} 12-\mathrm{H} 12$ & 119.9 \\
\hline $\mathrm{C} 7-\mathrm{C} 6-\mathrm{H} 6 \mathrm{~A}$ & 108.9 & $\mathrm{C} 7-\mathrm{C} 12-\mathrm{H} 12$ & 119.9 \\
\hline $\mathrm{C} 2-\mathrm{N} 1-\mathrm{C} 1-\mathrm{C} 4$ & $-0.2(3)$ & $\mathrm{S} 1-\mathrm{C} 1-\mathrm{C} 4-\mathrm{C} 5^{\mathrm{i}}$ & $-0.3(4)$ \\
\hline $\mathrm{C} 6-\mathrm{N} 1-\mathrm{C} 1-\mathrm{C} 4$ & $175.6(2)$ & $\mathrm{N} 1-\mathrm{C} 1-\mathrm{C} 4-\mathrm{C} 3$ & $-1.2(3)$ \\
\hline $\mathrm{C} 2-\mathrm{N} 1-\mathrm{C} 1-\mathrm{S} 1$ & $-178.89(19)$ & $\mathrm{S} 1-\mathrm{C} 1-\mathrm{C} 4-\mathrm{C} 3$ & $177.4(2)$ \\
\hline $\mathrm{C} 6-\mathrm{N} 1-\mathrm{C} 1-\mathrm{S} 1$ & $-3.1(3)$ & $\mathrm{C} 4-\mathrm{C} 3-\mathrm{C} 5-\mathrm{C} 4^{\mathrm{i}}$ & $-0.3(4)$ \\
\hline $\mathrm{C} 1-\mathrm{N} 1-\mathrm{C} 2-\mathrm{C} 3$ & $1.5(3)$ & $\mathrm{C} 2-\mathrm{C} 3-\mathrm{C} 5-\mathrm{C} 4^{\mathrm{i}}$ & $-180.0(2)$ \\
\hline
\end{tabular}




$\begin{array}{llll}\mathrm{C} 6-\mathrm{N} 1-\mathrm{C} 2-\mathrm{C} 3 & -174.3(2) & \mathrm{C} 2-\mathrm{N} 1-\mathrm{C} 6-\mathrm{C} 7 & -108.7(3) \\ \mathrm{C} 1-\mathrm{N} 1-\mathrm{C} 2-\mathrm{S} 2 & -177.3(2) & \mathrm{C} 1-\mathrm{N} 1-\mathrm{C} 6-\mathrm{C} 7 & 76.0(3) \\ \mathrm{C} 6-\mathrm{N} 1-\mathrm{C} 2-\mathrm{S} 2 & 7.0(4) & \mathrm{N} 1-\mathrm{C} 6-\mathrm{C} 7-\mathrm{C} 12 & 64.7(3) \\ \mathrm{N} 1-\mathrm{C} 2-\mathrm{C} 3-\mathrm{C} 5 & 177.5(3) & \mathrm{N} 1-\mathrm{C} 6-\mathrm{C} 7-\mathrm{C} 8 & -116.7(3) \\ \mathrm{S} 2-\mathrm{C} 2-\mathrm{C} 3-\mathrm{C} 5 & -3.8(4) & \mathrm{C} 12-\mathrm{C} 7-\mathrm{C} 8-\mathrm{C} 9 & 0.0(4) \\ \mathrm{N} 1-\mathrm{C} 2-\mathrm{C} 3-\mathrm{C} 4 & -2.2(3) & \mathrm{C} 6-\mathrm{C} 7-\mathrm{C} 8-\mathrm{C} 9 & -178.7(3) \\ \mathrm{S} 2-\mathrm{C} 2-\mathrm{C} 3-\mathrm{C} 4 & 176.5(2) & \mathrm{C} 7-\mathrm{C} 8-\mathrm{C} 9-\mathrm{C} 10 & 0.1(5) \\ \mathrm{C} 5-\mathrm{C} 3-\mathrm{C} 4-\mathrm{C} 5 & \mathrm{C} 8-\mathrm{C} 9-\mathrm{C} 10-\mathrm{C} 11 & -0.3(5) \\ \mathrm{C} 2-\mathrm{C} 3-\mathrm{C} 4-\mathrm{C} 5^{\mathrm{i}} & 0.3(4) & \mathrm{C} 9-\mathrm{C} 10-\mathrm{C} 11-\mathrm{C} 12 & 0.4(5) \\ \mathrm{C} 5-\mathrm{C} 3-\mathrm{C} 4-\mathrm{C} 1 & -180.0(2) & \mathrm{C} 10-\mathrm{C} 11-\mathrm{C} 12-\mathrm{C} 7 & -0.3(5) \\ \mathrm{C} 2-\mathrm{C} 3-\mathrm{C} 4-\mathrm{C} 1 & -177.6(2) & \mathrm{C} 8-\mathrm{C} 7-\mathrm{C} 12-\mathrm{C} 11 & 0.1(4) \\ \mathrm{N} 1-\mathrm{C} 1-\mathrm{C} 4-\mathrm{C} 5^{\mathrm{i}} & 2.1(3) & \mathrm{C} 6-\mathrm{C} 7-\mathrm{C} 12-\mathrm{C} 11 & 178.8(3)\end{array}$

Symmetry code: (i) $-x+2,-y+1,-z$.

Hydrogen-bond geometry $\left(A,{ }^{\circ}\right)$

\begin{tabular}{lllll}
\hline$D-\mathrm{H} \cdots A$ & $D-\mathrm{H}$ & $\mathrm{H} \cdots A$ & $D \cdots A$ & $D-\mathrm{H} \cdots A$ \\
\hline $\mathrm{C} 6-\mathrm{H} 6 B \cdots \mathrm{S} 2$ & 0.99 & 2.75 & $3.208(3)$ & 109 \\
$\mathrm{C} 6-\mathrm{H} 6 B \cdots C g 1^{\mathrm{ii}}$ & 0.99 & 2.66 & $3.498(3)$ & 142 \\
\hline
\end{tabular}

Symmetry code: (ii) $x, y+1, z$. 